\title{
RAPIDLY PROGRESSIVE CEREBRAL DEGENERATION (SUBACUTE VASCULAR ENCEPHALOPATHY) WITH MENTAL DISORDER, FOCAL DISTURBANCES, AND MYOCLONIC EPILEPSY
}

BY

\author{
D. P. JONES and S. NEVIN \\ From The Maida Vale Hospital for Nervous Diseases, London
}

The two cases which form the subject of this paper presented clinical signs of a subacute degenerative process affecting the cerebral cortex. The mechanism of the pathological changes is obscure but we have, for reasons which will be discussed, formed the opinion that vascular insufficiency is the important factor in their causation. The patients presented clinical features which were on the whole very different from those well recognized as characteristic of cerebrovascular disease, and from such acute manifestations of this disease as hypertensive encephalopathy. Also, in may respects the pathological findings are not those typical of cerebral arteriosclerosis. A few cases have been described in the literature which resemble them, but in none of these has vascular insufficiency been advanced as an explanation of the pathological changes. The relevant literature will therefore be referred to in the discussion of the pathology.

\section{Case Reports}

Case 1.-A. C., a housewife aged 66, was admitted to Maida Vale Hospital for Nervous Diseases, under the care of Dr. Dimsdale, on December 8, 1951. She died five weeks later on January 12, 1952.

The patient had been in her usual state of health until 10 weeks before her admission when she became easily fatigued and short of breath, giddy with slight unsteadiness in walking, and subject to double vision at times, particularly in the mornings. She was occasionally forgetful, but no other mental changes were noted until three weeks before admission when she was taken into the Royal Free Hospital for observation. There she quite suddenly became confused, and within a few days was unable to recognize her son except for brief moments. Her condition grew worse; she became restless, noisy, and resistive and was transferred to the Maida Vale Hospital for neurological investigation.

Clinical Examination.-On admission the patient was restless, grimacing, shouting or talking, and was quiet for brief periods only. She seemed at times hallucinated and spoke as if to domestic animals in the room. She was for the most part uncooperative and only occasionally would she obey simple commands. Passive movements were resisted, sometimes with a facial expression of resentment. She appeared quite disorientated for time and place but the delirious state precluded detailed investigation. She was incontinent of urine and faeces. The optic discs were normal but the retinal arteries showed moderate arteriosclerosis. A threat produced a blink reflex from both halves of the visual fields. The pupils were equal and reacted to light; the eye movements appeared to be full. She moved her limbs freely and no motor abnormality was manifest. The deep reflexes were present and symmetrical ; the abdominal reflexes were not elicited but both plantar reflexes were flexor. Sensation could not be tested adequately but she appeared to appreciate painful stimuli applied to either side. The blood pressure was $140 / 90 \mathrm{~mm}$. $\mathrm{Hg}$ and the radial arteries were slightly thickened. The heart was not enlarged and no abnormality was found on clinical examination of the chest and abdomen.

The urine, on routine examination, was normal as was the blood count. There was no stippling or abnormality of the erythrocytes although pernicious anaemia had been diagnosed in 1943, since when she had received regular liver injections. The Wassermann reaction was negative in the blood and cerebrospinal fluid. The latter was under normal pressure and contained no abnormal constituents. Radiographs of the skull and ventriculograms were normal.

Progress.-The patient gradually grew worse in hospital and, four days after admission, she no longer spoke coherently although remaining noisy and hyperactive. At this time myoclonic jerks were first observed. They affected mainly the face, eyes and upper limbs but at times involved the whole trunk. They varied in severity from brief twitches not readily seen to large movements affecting an entire limb or the whole trunk. They occurred irregularly at intervals of a second or so for up to half a minute at a time but lessened in frequency when the patient was quiet, and were absent for a time following sedation with barbiturates or paraldehyde. They did not 
appear to be influenced by epanutin or by tridione. An electroencephalographic (E.E.G.) examination was first performed at this time and showed a remarkable pattern of high voltage discharges synchronous with myoclonic jerks though occurring also without visible myoclonus. A more detailed analysis of the E.E.G. changes is given below.

At the time of onset of the myoclonic jerking a periodicity in the patient's behaviour was noticed. She would at one time be quiet with the eyes more often closed than open, the arms flexed across the chest, the hands tightly clenched and the feet plantar-flexed. After one to two minutes in this state a single cry would often usher in a noisy, restless period lasting for about 30 seconds during which she flushed, sweated profusely, and breathed more deeply. Coincidentally the myoclonic jerks increased noticeably in frequency. A tachycardia with elevation of the blood pressure up to $200 / 120 \mathrm{~mm}$. $\mathrm{Hg}$ was sometimes, but not always, observed during these excitable periods.

Three weeks after admission the periodicity in behaviour became less marked, the patient being more drowsy with gradual lessening of spontaneous motor activity and utterance of any kind. Myoclonic jerks continued to occur though likewise less frequently ; they could at times be provoked by a pin-prick applied to a limb. Sometimes there occurred brief attacks in which the head and eyes turned upwards and to the right in association with a few clonic jerks of the limbs for two to four seconds. Sometimes these jerks were predominantly right-sided and slight weakness of the right lower face and spasticity of the right arm were noted at this time. No major generalized seizures were observed at any time.

A week before death the patient had become immobile and deeply comatose with bilateral extensor plantar responses. Myoclonic jerks were infrequent. The pulse rate, at first varying, now showed a persistent tachycardia and Cheyne-Stokes respiration occurred intermittently. For two days before death, which took place seven weeks after the onset of the acute mental disturbance, the patient was in a profound coma and unresponsive to any form of stimulus. The myoclonic jerks had ceased.

E.E.G. Observations.-On December 13, five days after admission, the first E.E.G. recording was made (Fig. 1). It showed an unusual pattern of polyphasic high-voltage complexes, synchronous in time in all areas and occurring in long runs at a frequency of about 1 per second with a duration of $\frac{1}{4}$ to $\frac{1}{2}$ second. Separating such runs were periods of lower voltage rhythmical or irregular slow-wave activity, the demarcation between the two patterns being fairly abrupt and clearly apparent. The high voltage complexes varied greatly in shape, having sometimes the appearance of triphasic sharp waves and at others consisting of more sinusoidal activity. The complex recorded from a particular area might vary in

(2)
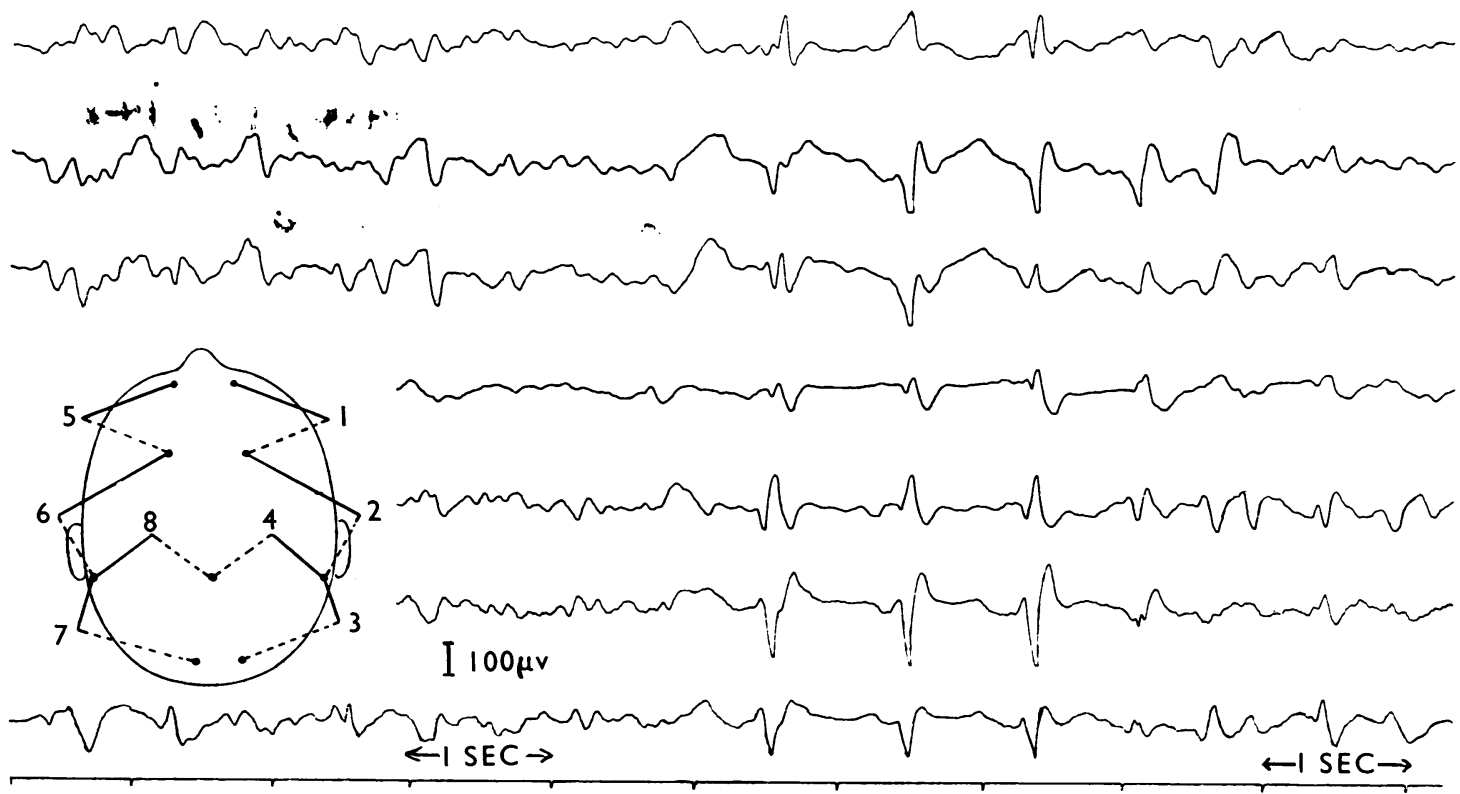

FIG. 1.-Case 1 : initial E.E.G. recording showing a run of polyphasic complexes following upon a period of lower-voltage activity. 
shape from moment to moment but sometimes a particular form would recur or would alternate with one of different outline. Often homologous areas of the brain gave rise to complexes closely resembling each other. The amplitude varied considerably from place to place and moment to moment ; sometimes it was highest at the beginning of a run and at other times showed an irregular recruitment. Myoclonic jerks were often observed to coincide with cortical discharges though bearing no apparent relationship to their amplitude or configuration. Many complexes were present, however, without visible myoclonic jerks.

Afferent stimuli of various kinds (Fig. 2) almost invariably effected a temporary inhibition of the cortical discharges, leading either to their cessation or to a clear decrease in amplitude. Repetitive stimuli were not effective in this respect other than at the start of the stimulus period. Within three weeks of admission the E.E.G. had shown a gradual change in that complexes tended at times to be more widely spaced. During involuntary hyperpnoeic periods, however, complexes occurred again at a frequency of 1 per second, continuing so until the hyperpnoea abated and these were not subject to blocking by afferent stimuli. Sometimes complexes were replaced by trains of slow waves lasting one to two seconds with relatively flat periods intervening, and then, after a more pronounced low amplitude phase, the succeeding discharge would again appear as a complex. During the week before death discharges were clearly occurring at greater intervals, and for long periods only bursts of rhythmical slow activity would occur (Fig. 3). High-voltage complexes were, however, still present episodically at the last recording 60 hours before death.

Pathological Findings in Case 1.-At necropsy little of note was found except in the brain. There was minimal atheroma of the aorta and coronary vessels but the radial arteries were tortuous and sclerotic, showing microscopically moderate thickening of the media and some intimal proliferation. Renal arterioles were also thickened to a slight extent. There was no abnormality of the pancreas, adrenals, stomach, or other viscera. Apart from moderate atheroma of the basilar artery the brain presented a normal external appearance and weighed 1,230 g. It was removed entire and after fixation sectioned vertically, material for histological examination being taken from all representative areas.

Cerebral Cortex.-In Nissl preparations the cortical layers were not grossly disorganized but everywhere two pathological changes were evident : first, small areas of status spongiosus and, secondly, some general thinning of the cortical neurons, especially in the deeper layers. The first change was the more prominent and was present in all regions of the cortex examined. It varied greatly in severity from isolated foci to a fairly widespread lesion extending through all layers (Fig. 4). In the frontal cortex only one small focus might be found in any convolution, usually in layers 4 and 5 , but at some points
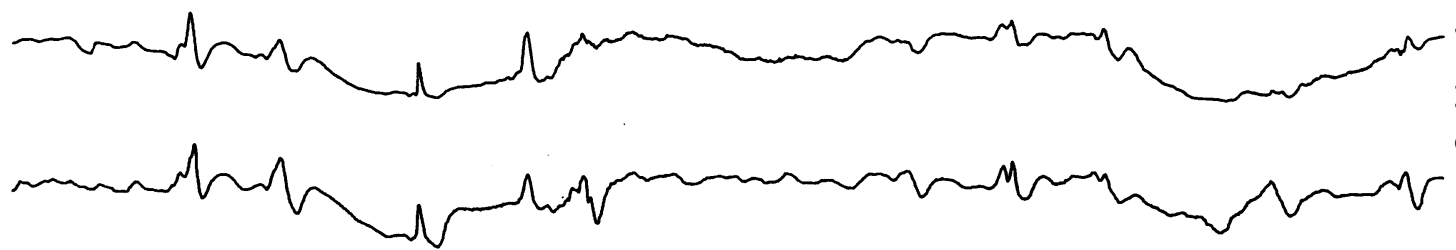

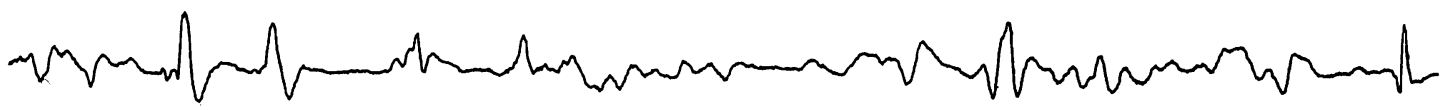

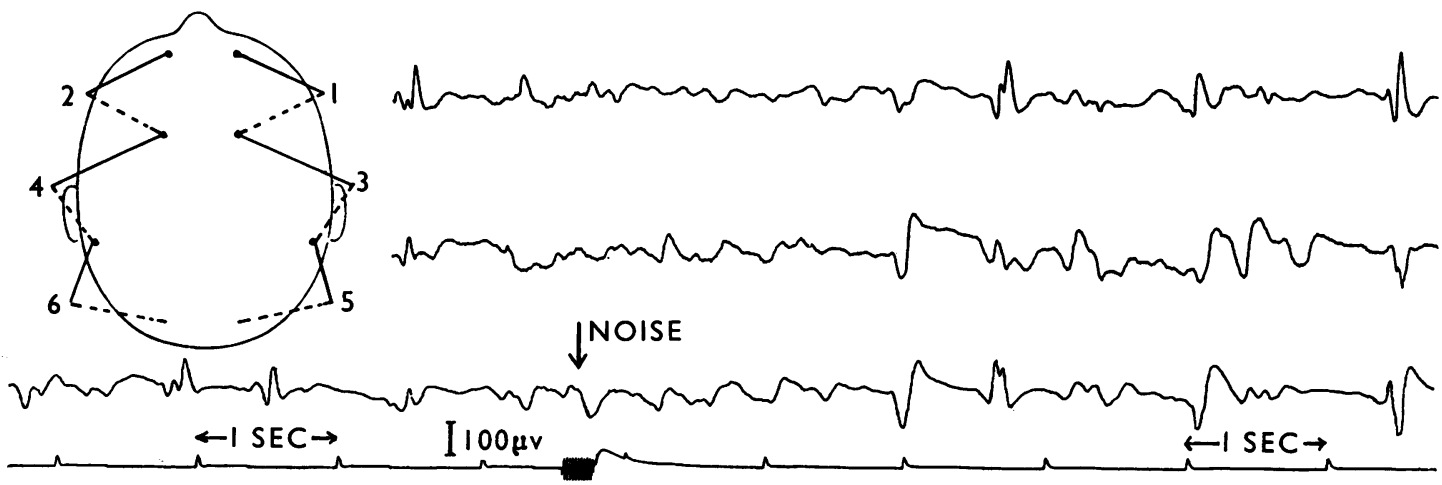

FIG. 2.-Case 1 : sample of E.E.G. showing the inhibitory effect of an acoustic stimulus. 

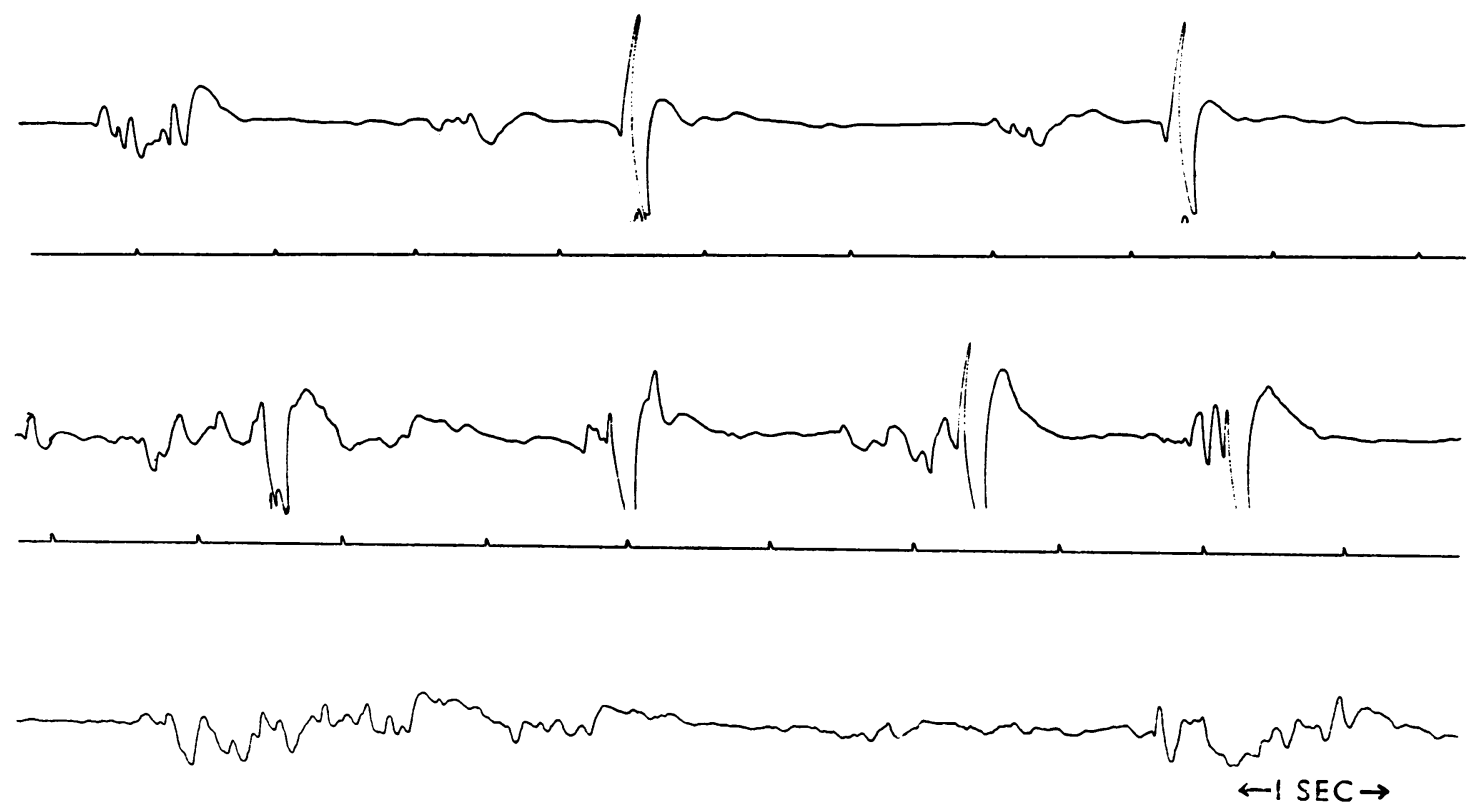

FIG. 3.-Case 1 : recording from brain surface three days before death to show the slower repetition rate of complexes and their replacement at times by trains of slow waves (diphasic sharp-wave in both trace measures/millivolt).

numerous foci of varying size occurred together at the bottom or along the sides of a sulcus.

The condition was on the whole more marked centrally than anteriorly, this being especially so in the upper extent of the superior frontal gyrus where all layers of the cortex were involved. The foci were irregularly oval and elongated in the vertical axis of the cortex ; at some points they coalesced and were surrounded by paling or loss of nerve cells. The post-central gyrus was slightly more affected than the precentral and, still more posteriorly, severe changes were observed in the parietal cortex and superior lip of the Sylvian fissure with predominant involvement of layers 2,3 , and 4 . In the temporal lobe the changes were everywhere slight except at the inferior margin of the fissure of Sylvius. No spongiose lesions were seen in Ammon's horn. In the occipital lobe only one or two small isolated lesions were seen in the striate area but in the parastriate region quite large areas of status spongiosus were visible and were especially found at the depths of the sulci. Severe lesions were also found elsewhere in the occipital lobe.

Under high magnification the spongiose lesions appeared as irregular clusters of empty spaces between which strands of ground substance were interwoven. These strands, which stained slightly yellow with van Gieson, contained sometimes isolated neuroglial and oligodendroglial nuclei and, more rarely, faintly staining nerve cells or cell fragments, but otherwise there was complete destruction of parenchymal and ground substance in the area of the lesion. In the tissue adjacent to spongiose areas occasional nerve cells were seen in various stages of dissolution (Fig. 5). Fragments of neurons could be recognized by faintly staining granules and there were also complete cell shadows containing sometimes a swollen and vesicular nucleus. Triangular cell forms were occasionally seen. Though maximal in tissue adjacent to spongiose areas, the neuronal changes were diffusely present in varying degree in all regions of the cortex examined. There was some irregular loss of Betz cells in the motor cortex but, in Ammon's horn, neither in the Sommer's sector nor in the end folium was any gross loss of cells to be seen although some cytolysis of neurons had taken place.

A striking feature was the proliferation of neuroglial cells. These were swollen with a large, faintly granular nucleus and irregular cell body often containing, in the Nissl preparation, dark green granules of considerable size. Neuroglial cells with two or three nuclei were seen. The neuroglial reaction extended into intact cortex as well as being present adjacent to areas of status spongiosus; it involved all layers but especially the molecular and outer cortical layers (Fig. 6) where it was associated with considerable gliosis. In regions of the cortex where neuronal changes were most severe regressive changes were also present in the neuroglia. There was some microglial proliferation, best seen in the molecular layer, and here it was difficult to discern nerve cells amongst the proliferated glia. Bielschowsky staining 


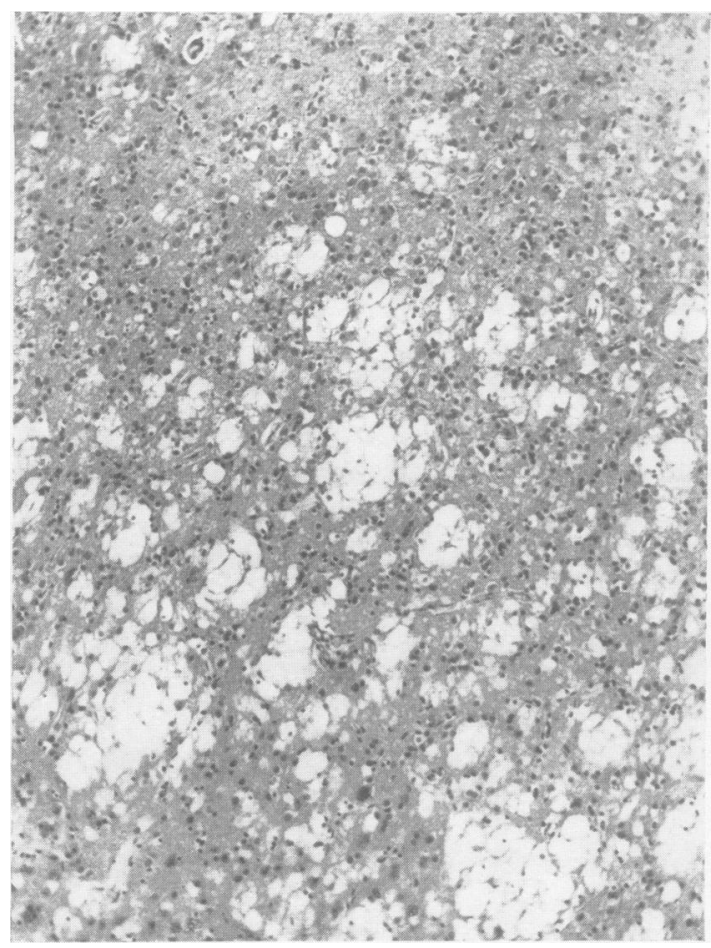

Fig. 4.-Case 1 : central cortex stained with haematoxylin and eosin $\times 95$ to show status spongiosus involving the deeper cortical layers.

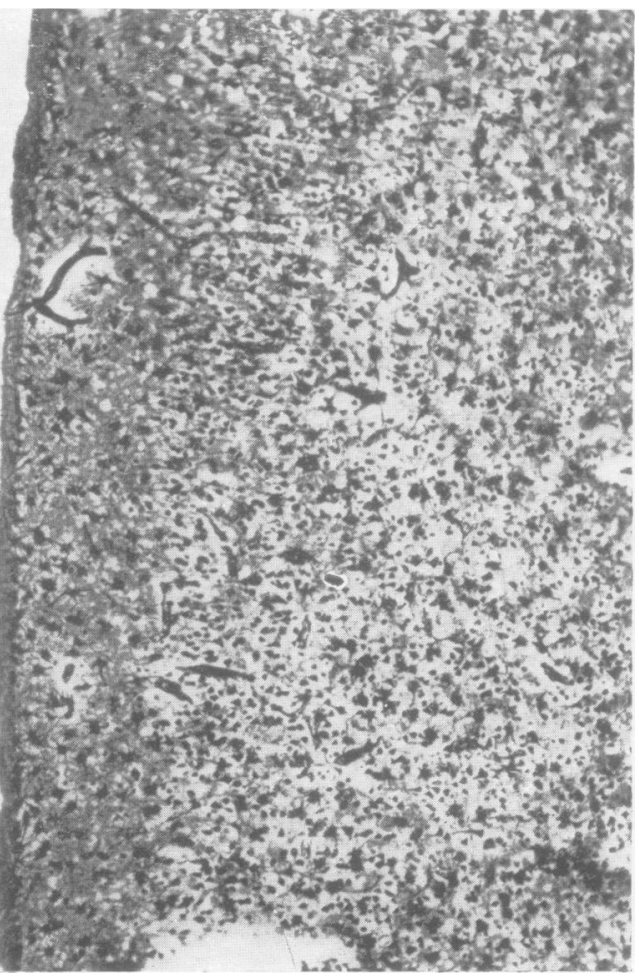

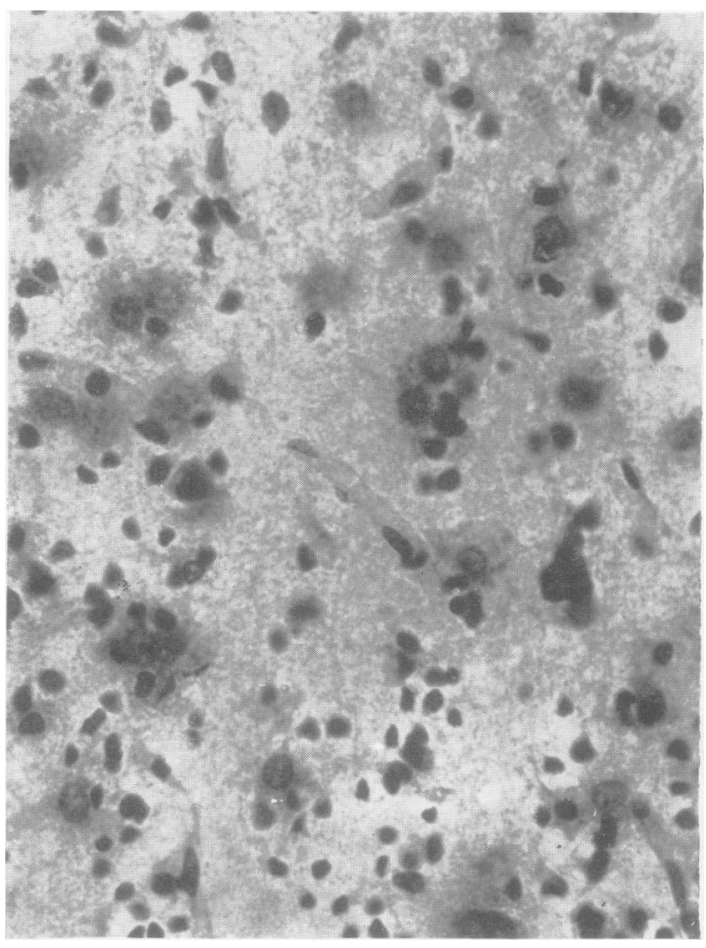

Fig. 5.-Case 1: frontal cortex stained with Nissl $\times 280$ to show loss and degeneration of neurons, some of which are not easily differentiated from the proliferated neuroglial in the Nissl preparation.

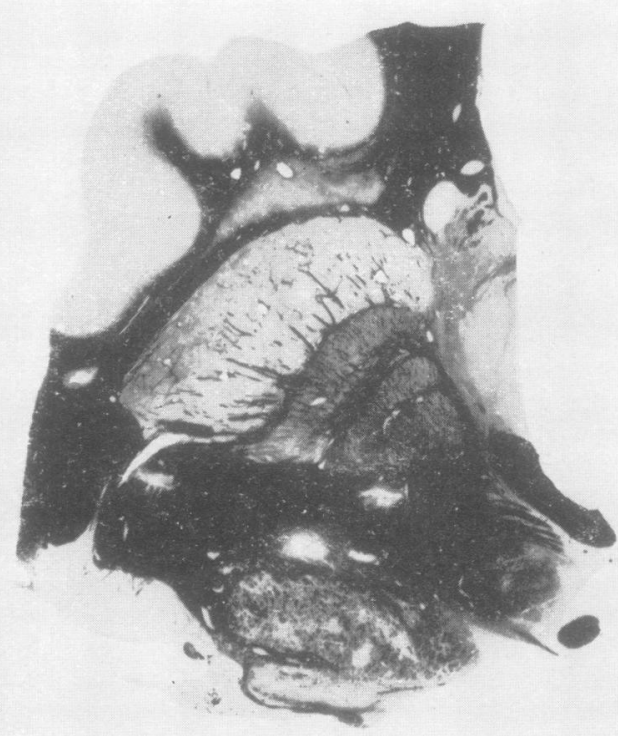

FIG. 6.-Case 1: frontal cortex stained with Hortega's silver carbonate for astrocytes 95 . The diffuse astroglial reaction is illustrated.

FIG. 7.-Case 1: section through the basal ganglia nuclei (Heidenhain) showing softenings and état criblé. 
showed diminution of cell and fibre content of the cortex but this method was not very successful as the brain was not removed until 26 hours after death.

In myelin preparations the status spongiosus, seen with Nissl staining, was very apparent, and radiating fibres were displaced or disrupted. Apart from the spongiose areas, scattered irregularity or interruption of fibres could be seen, and tangential fibres in the molecular layer were sometimes thin and fragmented. The white matter underlying the cortex everywhere stained darkly and no pathological changes were detected.

In the cerebral cortex and subjacent white matter pathological changes in vessels were minimal ; the larger cortical vessels were for the most part intact although there was a good deal of sclerosis of small arterioles, especially in the molecular layer and also to some extent in the white matter where some perivascular dilatation was often observed. Stains for reticulum showed at many points irregular and quite thick reticulum strands or networks spreading along the capillaries, but this was not specifically correlated with the areas of status spongiosus where apparently intact capillaries were also to be seen. There was some leptomeningeal thickening with a moderate amount of hyaline degeneration in surface vessels.

Basal Ganglia.-The most significant pathological change was the occurrence of small softenings, generally oval and a few millimetres in length (Fig. 7). These were present in the basal ganglia, the internal capsule, and the central corpus callosum. They were for the most part complete, showing only a small amount of tissue debris and fat granule cells and surrounded by an irregular zone of neuroglial and fibroblastic proliferation with much new vessel formation. In the caudate nucleus and putamen one or two foci of status spongiosus similar to that present in the cortex were seen and, close to them, some cytolysis of neurons and considerable neuroglial proliferation. In the globus pallidus there was some loss of large cells with overgrowth of neuroglial cells showing large nuclei and green or yellow-green granules in the cytoplasm with the Nissl stain.

Softenings were also apparent in the thalamus, several being located medially close to the ventricular surface. Apart from the softenings the thalamus showed diminution of nerve cells and areas of paling without status spongiosus, and there was also moderate neuroglial and slight microglial overgrowth. A striking feature in the basal ganglia region was the considerable dilatation of perivascular spaces to the extent of producing the appearance of état criblé, particularly in the lenticular nucleus, claustrum, and external capsule. In a few of the larger blood vessels, clear fibrous tissue hyperplasia into the enlarged surrounding space had occurred with some lymphocytic infiltration. The majority of spaces, however, especially those surrounding the smaller vessels, were empty. Despite the enlarged perivascular spaces, gross changes in the vessel walls were rare and hyaline degeneration was seen only occasionally in small vessels.

Brain-stem.-In the midbrain, pons, medulla, cerebellum, and upper cervical cord no significant patho$\mathrm{E}^{*}$ logical changes were seen apart from a slight outfall of cells in the olivary and dentate nuclei with some neuroglial proliferation. There was a slight loss of Purkinje cells in some of the cerebellar folia with neuroglial overgrowth in the molecular layer as well.

Case 2.-A. L., a male clerk aged 62, was admitted to Maida Vale Hospital on December 19, 1951, under the care of Dr. Dimsdale, and died on January 19, 1952.

There was no history of any significant illness in the past and he had been well until 10 weeks before admission when he noticed slight stiffness of the right arm with a pins-and-needles sensation down its outer aspect, followed within a week by weakness of the limb and unsteadiness on attempted use. Two weeks later, pins and needles were felt in the right leg associated with slight weakness in walking and a tendency to fall to the right. At the same time he noticed difficulty in enunciating words. During the period before entry to hospital weakness of the right side, especially of the arm, slowly progressed and the paraesthesiae were replaced by a constant feeling of numbness.

On admission he was alert, normally orientated, and showed no signs of mental deterioration on systematic testing. He was dysarthric but not dysphasic although right-handed. There was weakness of the right face, arm and leg, most marked in the arm. Spasticity was noticeable only in the arm but the tendon jerks were abnormally brisk in both arm and leg; the abdominal reflexes were depressed on that side and the right plantar response was extensor. There was loss of postural and vibration sensibility in the arm and, to a lesser degree, in the leg, and two-point discrimination was defective in the right hand. Superficial sensibility was preserved but there was a slight over-reaction to painful stimuli on the right. The visual fields were full and there was no defect of visual attention. A slight nystagmus was present on turning the eyes to the left or upwards. On the left side no weakness or gross sensory loss was found but twopoint discrimination was slightly impaired in the left hand. Clinical examination of the heart, lungs, and abdomen revealed no abnormality ; the blood pressure was $120 / 80$ $\mathrm{mm}$. $\mathrm{Hg}$ and there was no significant thickening of the peripheral arteries.

Progress.-The patient's condition deteriorated steadily. During the first week dysarthria became more marked and the right hemiplegia more severe, spasticity of the arm giving place to flaccidity; at times occasional, slow, involuntary movements of the hand took place. The right side became painful to touch or on passive movement. Two weeks after admission spasticity had developed on the left side and at this time myoclonic jerks were first seen. They occurred in episodes lasting several minutes and affected either arm though more prominently the right. The patient remained conscious but slow to respond and grossly dysarthric.

During the two weeks before death paralysis involved all four limbs and the face bilaterally. The patient was drowsy, doubly incontinent, and speechless. Myoclonic jerks of the upper limbs continued, remaining more frequent on the right side Three days before death the 
patient would still open his eyes on command but there was by then a complete bilateral flaccid weakness with areflexia. Myoclonic jerking continued though now less frequently. Fever with Cheyne-Stokes respiration, a fall in blood pressure, and peripheral cyanosis supervened just before death.

Left carotid arteriography on December 21 showed a normal arterial and venous pattern but with a $5 \mathrm{~mm}$. shift of the anterior cerebral artery to the right. Air studies a week later did not show this asymmetry, the ventricular system being normal. The cerebrospinal fluid, examined on December 28, 1951, was at a pressure of $170 \mathrm{~mm}$. of water and contained $50 \mathrm{mg}$. of protein per $100 \mathrm{ml}$. with 8 lymphocytes per c.mm. (An examination had been performed elsewhere 10 days before with the finding then of a pressure of $100 \mathrm{~mm}$., protein $120 \mathrm{mg}$. per $100 \mathrm{ml}$. and no cell increase.) The urine on routine examination was normal, the blood haemoglobin $105 \%$, and the Wassermann reaction negative in blood and cerebrospinal fluid.

E.E.G. Findings.-The E.E.G. recorded on the day of admission showed a low-voltage pattern with irregular runs of slow activity occurring bilaterally. These were most prominent in the frontal region and sometimes more marked on the left side. On the day prior to death bilateral complexes, usually triphasic, were seen (Fig. 8). They would be present for periods lasting $10-15$ seconds separated by runs of rather rhythmical slow activity. Sometimes the complexes contained spikes; they were temporarily blocked by afferent stimuli. No myoclonic jerks were observed during the recording.

Pathological Findings in Case 2.-A post-mortem examination was performed three hours after death. A moderate degree of aortic and coronary atheroma was found but the heart was not enlarged and there was no evidence of degenerative change in the myocardium. The pancreas, kidneys, adrenals, and other viscera were histologically normal.

The brain weighed $1,570 \mathrm{~g}$. but in the occipital and frontal polar regions the cortical surface appeared moderately atrophic with widening of the sulci. Vertical sections revealed slight congestive changes in the white matter but no other gross lesions.

Cerebral Cortex.-The cerebral cortex showed two definite pathological changes, widespread but nowhere of exceptional severity. These were general thinning of the cortical layers, and neuroglial proliferation (Figs. 9 and 10). The latter was most marked in the outer granular and pyramidal layers, giving at some points an appearance of increased cellularity in these regions. Cell loss, on the other hand, was of greatest severity in the deeper layers. Nowhere was there gross disorganization of the cortex and, although sections were taken from many regions, at only one or two points was a minimal and circumscribed cavitation of the ground substance observed. In the deeper layers of the cortex some light-staining remains of degenerated nerve cells were visible at many points ;
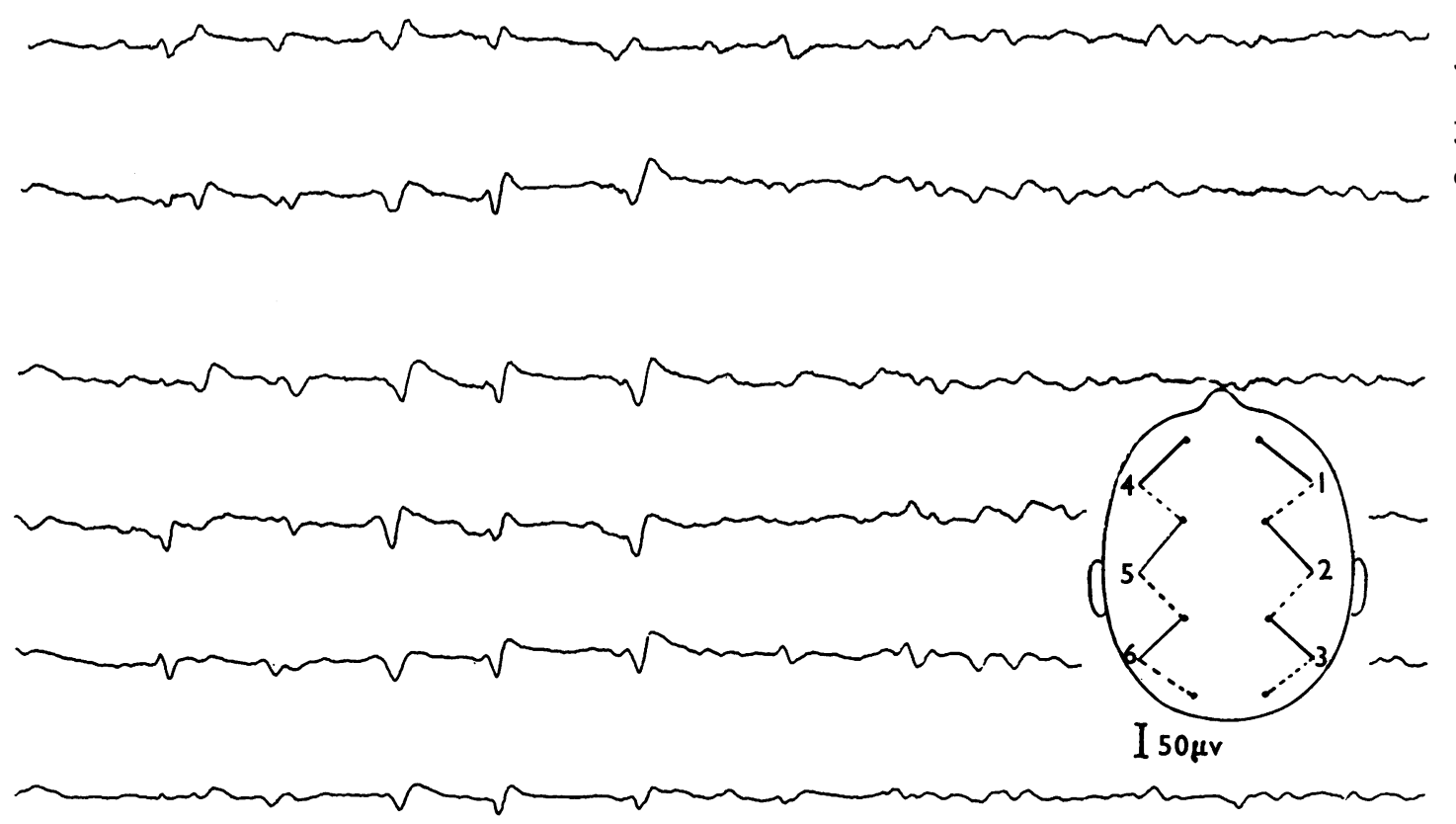
FiG. 8. -Case 2: E.E.G. recorded on the day before death illustrating the pattern of recurrent discharges separated by periods of 


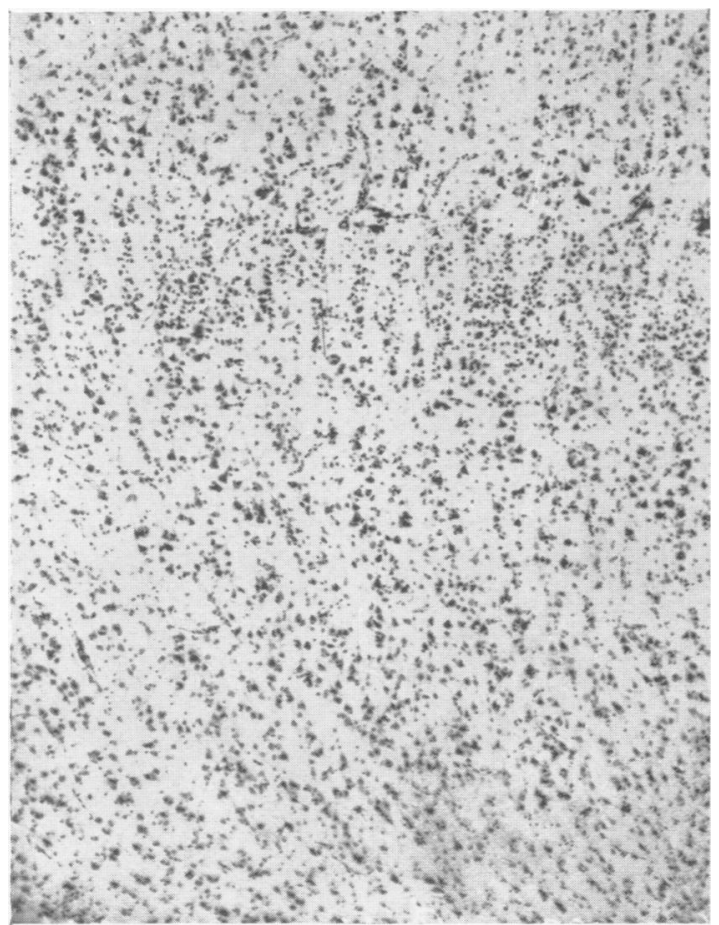

FIG. 9.-Case 2 : cortex in Island of Reil stained with Nissl $\times 95$ to show loss of nerve cells without gross disorganization of the cortical layers.

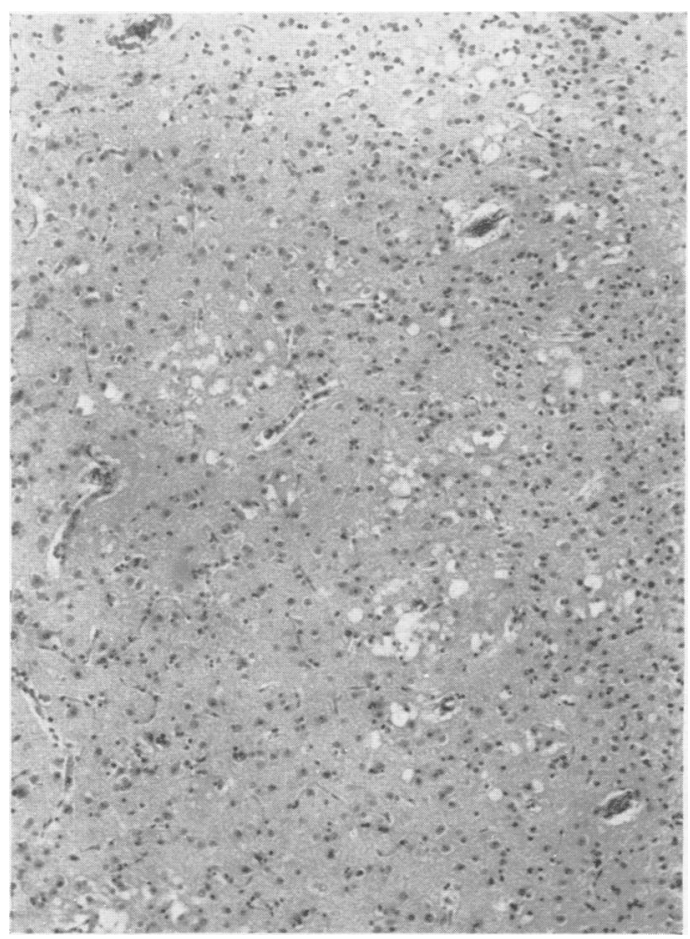

FIG. 11.-Case 2: occipital cortex stained with haematoxylin and eosin $\times 95$ to show slight status spongiosus in the inner layers.

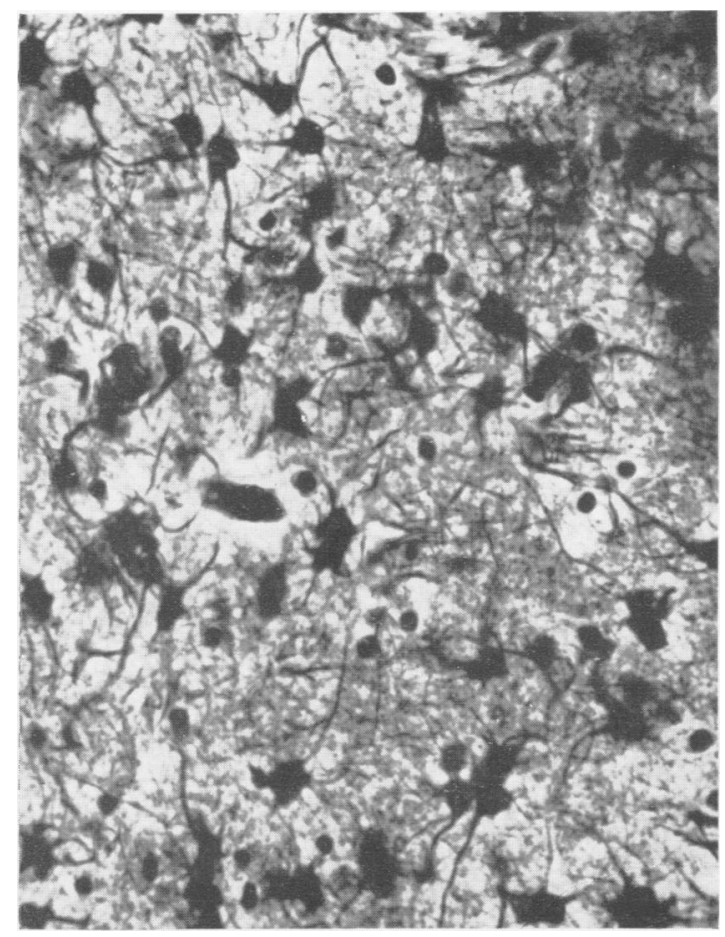

FIG. 10.-Case 2: parietal cortex stained with Hortega's silver carbonate for astrocytes $\times 280$ to show neuroglial proliferation in the deeper cortical layers.

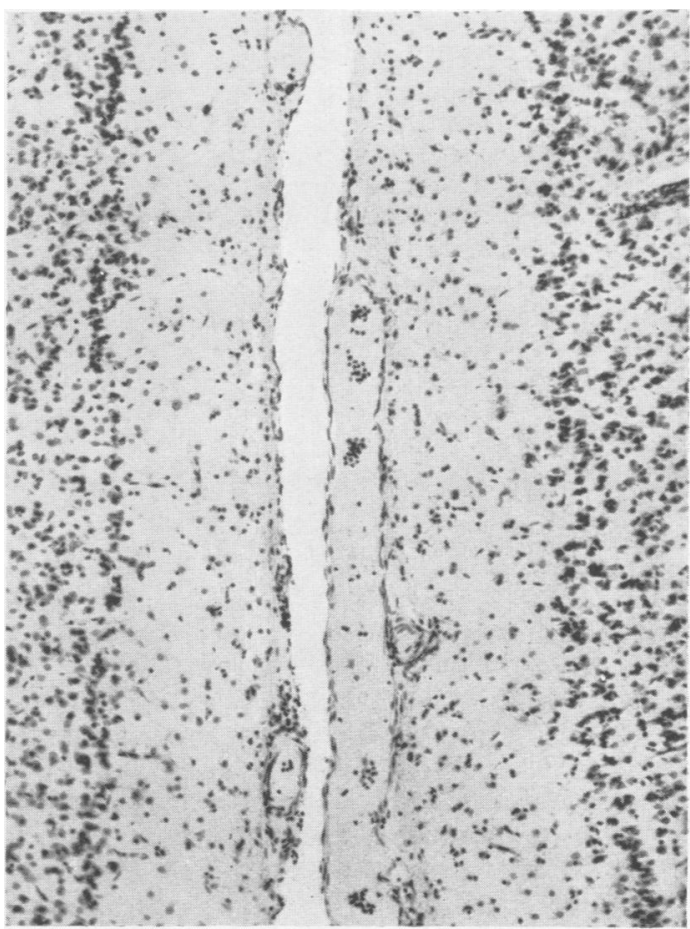

FIG. 12. Case 2: occipital cortex stained with Nissl $\times 95$ to show marked neuroglial overgrowth at the junction of the molecular and external granular layers. 
at others the nerve cells stained darkly and had shrunken or triangular nuclei, but typical examples of ischaemic or homogenizing cell change were not encountered. Many nerve cells in all layers contained fatty granules of varying number and size.

The proliferated neuroglial cells were enlarged and often the cell body was filled with granules. With Sharlach $\mathbf{R}$ these stained red like those in the nerve cells, and with Nissl stain greenish yellow. Often the cells were binucleate. Glial fibre stains revealed intense proliferation of glial fibres under the pia and in the molecular layer but also extending well into the outer cortical layers. There was a slight increase in microglia. Myelin preparations showed no significant change in the white matter nor the cortex.

In the frontal cortex the changes were present in all areas examined and tended to be more marked at the depths of some sulci. Neuroglial proliferation was moderate and fibrous gliosis involved mainly the molecular layer. In the central cortex the gliosis was more marked, with numerous binucleate cells in the outer layers. Some Betz cells had disappeared. In the temporal lobe the region of Ammon's horn was preserved and there was little reactive gliosis or significant thinning of neurons in Sommer's sector and the end folium. In other convolutions of the temporal lobe, however, the typical changes were everywhere present, and particularly so in the lower lip of the Sylvian fissure where considerable fibrous gliosis extended into the second and third layers. Here also, more than elsewhere, a slight tendency to a status spongiosus similar to that in Case 1 was present (Fig. 11).

In the occipital cortex the changes were as severe as in other regions and glial proliferation in the outer layers was especially marked. In one part of the area striata and adjacent parastriate region glial proliferation produced a sharp margin to the junction between the outer granular and molecular layers (Fig. 12).

Changes in the blood vessels were minimal apart from a moderate degree of pericapillary fibrosis, with some hyaline necrosis of the arterioles, especially in the molecular layer of the cortex. Here also, many small vessels in the deeper cortical layers were surrounded by considerably dilated perivascular spaces.

Subcortical Regions.-No significant changes were found in the basal ganglia, brain-stem, cerebellum or upper cervical cord, and there was no degeneration of the pyramidal tracts.

\section{Discussion}

E.E.G. Findings.-Although the E.E.G. study of the second case was limited it is clear that, in the later stage of the illness, the abnormalities recorded were similar to those in the first patient. Essentially these consisted of periodically recurring generalized complexes, varying in contour but often with sharpwave components, and remitting spontaneously or upon afferent stimulation. In the first case myoclonic jerks in different parts of the body were sometimes observed to coincide with the complexes although the occurrence of a jerk bore no apparent relationship to the shape of the discharge, being as often present with one of smooth outline as with one of sharp-wave form. Although this E.E.G. pattern is a very striking one it is not regarded as being in any way pathologically specific.

It is recognized that in subacute encephalitis recurrent complexes are found and that the E.E.G. may be of almost diagnostic distinctiveness (Radermecker, 1949 ; Radermecker and Macken, 1951 ; Cobb and Hill, 1950 ; Martin, Macken, and Hess, 1950). But in lipoidosis likewise (Cobb, Martin, and Pampiglione, 1952) a pattern of recurring discharges of varying shape and periodicity may be seen. Possibly then in these conditions, as well as in the cases here described, the E.E.G. abnormality is of a fundamentally similar nature, the periodic discharges arising as a result of severe, diffuse, cortical pathological change. The individual variations may depend upon the differing effects upon cortical neurons of local lesions and also upon the degree of general disturbance of brain function produced by the disease. In our cases pathological changes in the cortex were widespread and severe and it is easily understood that they could give rise to numerous discharging foci.

We have had the opportunity of observing the effect of $10 \%$ metrazol applied to the human cortex at a light plane of anaesthesia and have recorded recurring sharp-wave discharges followed at times by a slow-wave component, whereas, when anaesthesia is deepened, the spike is replaced by a train of slow waves. The similarity between the metrazol effect (previously observed by Johnson and Walker, 1952) and the discharges in our cases is evident, and it is of interest that in the first patient the sharp waves initially present tended to be replaced in the later stages of the illness by somewhat irregular but semirhythmic slow waves. It seems logical to infer that the cortical discharge was altered by deepening of the general cerebral disturbance as was the metrazol spike by the deepened anaesthesia and, in fact, pentothal intravenously in dosage of only $0.1 \mathrm{~g}$. was observed to suppress the spike component of the complexes occurring in the earlier stages of the illness.

The unspecific character of these E.E.G. patterns is further shown by the fact that somewhat similar discharges, occurring paroxysmally upon a lower voltage background, may be observed in many juvenile epileptic states, often caused by recognized pathological lesions of varied character. Although this is particularly the case with children, we have observed the phenomenon of recurrent generalized discharges in two adults who failed to improve after craniotomy, in the one case for a pituitary adenoma, 
and in the other for a frontal parasagittal meningioma. In both patients the state of consciousness was altered, and it is therefore inferred that, just as pentothal gives rise to periods of activity separated by intervals of low-voltage discharge (suppressionburst activity), so in pathological states the general effects leading to impairment of consciousness may condition the periodicity of the discharges. This may be through alteration of function of subcortical mechanisms, but it is possible that the cortical neurons, with their inherent tendency to rhythmic or periodic discharge, alone are involved.

We have therefore concluded that the E.E.G. pattern in our cases is best interpreted as signifying diffuse cortical discharges made synchronous and periodic by the pathological state of the cortical neurons and their interconnexions. Wesley Watson and Denny-Brown (1953), in discussing the general problem of myoclonic epilepsy occurring in a series of types of degenerative encephalopathies (subacute inclusion body encephalitis, amaurotic family idiocy), emphasize the unspecific character of the E.E.G. changes found in these disease states, changes which they consider as symptomatic of this whole class of disorder.

Pathological Changes.-Three features characterize the pathology in these cases : a widespread cortical degeneration with the formation of areas of status spongiosus, a diffuse neuroglial reaction in the cortex with considerable fibrous gliosis in the first three layers, and focal softenings in the basal ganglia and adjacent white matter. In the second patient changes were found only in the cortex and the status spongiosus formation was minimal, but there can be no doubt about the identity of the pathological process with that in Case 1. In interpreting these changes the presence of small focal softenings in the region of the basal ganglia in Case 1 is especially significant. These relatively recent softenings with irregular margins and surrounded by only a moderate degree of mesenchymal and neuroglial reaction can only be explained on the basis of a circulatory insufficiency in the small arterioles. Clinically and pathologically there is no evidence of any endogenous factor such as hypoglycaemia or of exogenous toxin that could give rise to them.

During clinical investigation of Case 1 several needle punctures of the brain were made, but it is improbable that trauma of this nature could have given rise to the numerous small softenings which were present and, moreover, careful examination of the brain showed that the needle tracks were not in relation to the softenings. A moderate dilatation of perivascular spaces in the region of the basal ganglia, with adventitial proliferation and some lymphocytic infiltration, also points to a primary vascular factor as the cause of the lesions.

It is not, however, easy to explain the mechanism of the vascular insufficiency in that sclerotic changes in the vessels are not so advanced as to make it likely that simple occlusion was the principal factor. A fairly widespread functional disturbance must have taken place to cause numerous softenings all presenting generally similar pathological features at necropsy. In the putamen there existed in the same section a well marked softening alongside a small area of status spongiosus, indicating a tissue reaction of different degree, most probably to the same kind of injury. There seems therefore good reason to regard the widespread cortical lesions as also being vascular in origin. Here again the sclerosis of the arterioles and the pericapillary fibrosis would appear inadequate to cause the lesion directly, while their widespread character is suggestive of a generalized functional disturbance of the circulation.

That a status spongiosus lesion in the cortex can arise on a vascular basis is well attested in the French literature. Lhermitte and Barrelet (1934) and Ameuille, Lhermitte, and Kudelski (1935) have observed it in air embolism, and Alajouanine, Hornet, and Thurel (1936) have described it in the cortex adjacent to haemorrhages in the white matter in three elderly patients, affecting in one instance the fifth and sixth cortical layers and in another the second cortical layer. These authors found, in the close proximity of the status spongiosus to the localized haemorrhage, clear proof of the vascular origin of the lesion which, they consider, may be due to circulatory damage to nerve cells, ground substance, or neuroglia with possibly hydration of the tissues. Status spongiosus has been a common finding in the brain of general paralytics especially in those with marked focal symptoms and convulsions, and here again Merritt and Springlova (1932), after a detailed study of the problem, conclude that the status spongiosus lesion is produced by the combination of a functional disturbance of the cerebral circulation with the inflammatory process. Bielschowsky (1919) discussing the same problem (status spongiosus in general paralysis of the insane) considered altered permeability of the cortical vessels leading to fluid accumulation in the tissues an important factor.

According to Spielmeyer (1922) a status spongiosus is indicative of an acute softening of the tissue (to be distinguished from the more usual vascular softening) in which the protoplasmic glia of the cortex and basal ganglia fails or is incapable of reacting to repair the defect. This concept may well be valid for 
the purely degenerative lesions of this type but it is probable that status spongiosus also occurs on a basis of vascular insufficiency, not necessarily complete but of a critical intensity, and associated with fluid exudation and impaired absorption. How this has been brought about in the cases described in this paper-where not only extensive spongiose lesions (especially in Case 1) were present, but also less intense tissue damage with focal cell loss and a widespread glial reaction approaching in form to gemästete glia-is the primary problem presented by this study. It is doubtful if it can be solved histopathologically. A functional impairment of cerebral circulation has been assumed and there is some clinical evidence to support this view in that, in the second case, the right-sided hemiplegia in the early stage of the illness was associated with some oedema of the left cerebral hemisphere as shown by the displacement of the anterior cerebral artery in the arteriogram. This suggests that the cerebral lesions had possibly resulted from vascular stasis and increased permeability of the vessels. Both patients were elderly with some degree of arteriosclerosis and it is probable that the defect is primarily a vascular one, although the possibility of some chemical or metabolic factor of endocrine or other origin acting on the vessels has to be borne in mind. For its existence, however, no clinical or pathological evidence is to hand. It seems very probable that further light on the problem may only be obtained by measuring the cerebral blood flow, and by a study of the reaction of cerebral vessels, in other similar cases, to those chemical and hormonal factors that are known to influence them.

The condition appears to be rare and we have not discovered in the literature comparable cases in which a vascular aetiology was thought to exist. Hallervorden (1930), however, under the title of "Peculiar and Non-classifiable Conditions", has described what appears to be a similar type of case. A 60-year-old woman died of erysipelas after an illness lasting three to four months characterized by mental disorder, hemianopia to the right, ideational apraxia, alexia, and excitability with perseveration in the utterance of meaningless words. Pathologically a fine status spongiosus, greatest in layers 2 and 4, was present over the whole cortex and affecting especially the posterior regions with nerve cell degeneration and neuroglial reaction in general similar to that present in the cases in this paper. Hallervorden regarded the condition as a purely degenerative process affecting the cerebral cortex. Fischer (1911) described a similar type of case in a 70-year-old man in whom the duration of symptoms was also four months. A sudden onset with focal symptoms pointing to the left temporal lobe had occurred in this case which showed, pathologically, focal atrophy in the left temporal region and a status spongiosus throughout the rest of the cortex.

Under the title " Concerning a Peculiar Organic Disease of the Presenium ", Heidenhain (1928) describes three cases which are all similar pathologically to those described in this paper. In the first patient aged 55 and in the second aged 53, both of whom died after an illness of 16 to 20 weeks, the prominent symptoms were cortical visual disturbance leading to blindness and profound mental changes characterized by excitability, clouding of consciousness, and disorientation. Gross palsies of one or other limb were not present but spasms of the whole musculature took place repeatedly.

In the third case the patient had been ill for a number of years but the terminal illness, characterized by rapid mental deterioration and epilepsy, may well have been a subacute encephalopathy of the type under discussion. Heidenhain regarded his cases as arising on a degenerative basis although he differentiates them pathologically from the degeneration which is found in Jakob Creutzfeld's syndrome and from other degenerative and inflammatory conditions. On account of the normality of the vessels he does not consider a vascular basis as possible, although he adopts the view that the status spongiosus is produced by severe exudation into the tissue spaces, as the myelin sheaths are clearly displaced by this particular lesion in some places. This view, if correct, points to some disturbance of circulatory function so that some vascular insufficiency as a cause of the nerve cell degeneration and neuroglial reaction is not an altogether impossible explanation of Heidenhain's cases.

There can be little doubt that these cases from the literature are similar pathologically to those described in this paper. We have considered a disorder of vascular function occurring in ageing and somewhat sclerotic cerebral vessels to be the most probable cause, but recognize that further study of the condition, clinically and pathologically, will be necessary to establish this, to elucidate further the mechanism of the vascular failure, and to determine whether or not altered capillary permeability is also a factor. Hallervorden (1930) referred to another case in the German literature in which an atrophic condition, progressive over some years, showed at necropsy a status spongiosus lesion of the cortex adjacent to a large atrophic area. He made the suggestion that a similar type of pathological process of more gradual development may underlie some of the rare and obscure focal atrophies in 
younger subjects. This suggestion appears to us well founded and points to the need for further histological study of biopsy material in such cases.

\section{Concluding Remarks}

The two patients whose case histories have been discussed in this paper, and the five described in the literature, appear to have suffered from a similar type of subacute encephalopathy. On the clinical side the analogous features are vague prodromal symptoms leading to a focal cerebral disorder such as hemiparesis, hemianopia, blindness, apraxia, or alexia associated with severe mental disturbance. Myoclonic epilepsy is a striking feature of the cases but may, in some instances, be absent unless we are incorrect in including in this type of encephalopathy the patient described in Hallervorden's article. We have put forward the view that there is at least a prima facie case for regarding the pathological changes as due to abnormal functioning of the cerebral vessels and consequent impaired nutrition of the cerebral cortex. On this basis it is proposed to refer to the cases as subacute vascular encephalopathy, a title which will distinguish them on the one hand from hypertensive encephalopathy, and on the other from the more slowly progressive states resulting from cerebral arteriosclerosis. If the vascular origin of the condition is eventually established then its clinical and pathological basis may eventually be considerably widened to include such cases of subacute encephalopathy as those described as vascular in origin by Alajouanine and van Bogaert (1950), cases somewhat different clinically and with more widespread pathological changes.

One final point worthy of empahasis is that the E.E.G. abnormalities, although in no way pathologically specific, may nevertheless prove helpful in diagnosis because of their rarity in adult life and because they indicate acute and generalized cortical disturbance. In a review of 100 cases of chronic cerebrovascular disease a similar pattern was not seen nor, to our knowledge, has it been described in degenerative conditions in this age group.

\section{Summary}

A clinical, electroencephalographic, and pathological description is given of two cases of a rapidly fatal illness in late adult life in which myoclonic epilepsy and progressive impairment of consciousness were striking features. The E.E.G. showed an unusual pattern of recurrent sharp-wave discharges over both hemispheres, and its significance in relation to the histological findings is discussed. In both patients death ensued within 15 weeks of the onset of the illness. In one there was a marked status spongiosus of the cortex, a general loss of cortical neurons, marked glial overgrowth, and numerous acute softenings in the basal ganglia. In the other, similar but less marked cortical changes were present. A vascular aetiology is postulated for the cerebral lesions, and for rapidly progressive cases of this type the name subacute vascular encephalopathy is suggested. Cases in the literature which are possibly similar are briefly discussed.

It is a pleasure to thank Dr. W. H. McMenemey for his help and advice in the preparation of this paper. We are also indebted to Mr. and Mrs. Gordon Cox, of the Pathological Department, and to Mr. Roberts and Miss Walker, of the E.E.G. Department, for their technical assistance. We are also grateful to Dr. Helen Dimsdale for permission to publish her cases.

\section{REFERENCES}

Alajouanine, T., Bogaert, L. van (1950). Rev. neurol. Paris, 82, 21. Alajot, T., and Thurel, R. (1936). Ibid., 65, 819. Ameuille, P., Lhermitte, J., and Kudelski, (1935). Ibid., 63, 867.

Bielschowsky, M. (1919). J. Psychol. Neurol., Lpz., 25, 72.

Cobb, W., and Hill, D. (1950). Brain. 73, 392.

Martin, F., and Pampiglione, G. (1952). Ibid., 75, 343.

Fischer, O. (191i). Z. ges. Neurol. Psychiat., 7, 1.

Hallervorden, J. (1930). In Handbuch der Geisteskrankheiten, ed. Bumke, O., vol. 11., p. 1094.

Heidenhain, A. (1928). Z. ges. Neurol. Psychiat., 118, 49.

Johnson, H. C., and Walker, A. E. (1952). Electroenceph. Clin. Neurophysiol., 4, 131 .

Lhermitte, J., and Barrelet (1934). Rev. neurol. Paris., II, 851.

Martin, F., Macken, J., and Hess, R. (1950). Schweiz. Arch. Neurol. Psychiat., 66, 217.

Merritt, H. H., and Springlova, M. (1932). Arch. Neurol. Psychiat., Chicago, 27, 987.

Radermecker, J. (1949). Acta neurol. psychiat. belg., 49, 222.

, and Macken, J. (1951). Rev. neurol., Paris, 85, 341.

Spielmeyer, W. (1922). Histopathologie des Nervensystems, vol. 1 , p. 335. Springer, Berlin.

Watson, C. Wesley, and Denny-Brown, D. (1953). Arch. Neurol. Psychiat., Chicago, 70, 151. 